\title{
Nicotine addiction and smoking cessation treatments
}

\author{
Jason Luty
}

It is estimated that $29 \%$ of adults in the UK smoke (Office for National Statistics, 2000). Each year smoking kills 120000 people (13 deaths per hour), making it the single most common preventable cause of death in Britain (Callum, 1998). Around half of all smokers will die prematurely because of their addiction (Peto et al, 1994). Their overall life expectancy is, on average, 8 years less than for nonsmokers (Callum, 1998). Smoking also costs the National Health Service (NHS) $£ 1500$ million, with around 1000 admissions every day for smokingrelated illnesses (Parrott et al, 1998). (However, the UK treasury earns $£ 8$ billion in tax on tobacco sold in the UK alone, in addition to the enormous revenue from overseas markets.)

Smoking is a major risk factor for at least 20 diseases, including coronary and peripheral vascular disease, chronic bronchitis and at least $80 \%$ of lung cancers (Callum, 1998). Blood coagulates in smokers more easily than in non-smokers, fibrinogen levels are higher and platelets are more likely to aggregate. These effects all contribute to thromboembolic diseases. Although nicotine itself is not carcinogenic, tobacco smoke contains over 200 other compounds that are potential carcinogens and smoking itself is the greatest single risk factor for lung cancer. Cigarette smoking significantly contributes to several other nasopharyngeal and upper gastrointestinal carcinomas (Benowitz, 1988).

\section{Is nicotine addictive?}

A 1988 US Surgeon General's report stated that nicotine is as addictive as drugs such as heroin and
Box 1 Addictive properties of nicotine in tobacco smoke

Compulsive use and craving

Tolerance (especially to adverse effects)

Withdrawal syndrome

Tendency to relapse after cessation

Use to relieve withdrawal symptoms

Persistent use despite evidence of harm

cocaine (Stolerman \& Jarvis, 1995). This represents the culmination of a gradual political shift from viewing smoking as a bad habit to seeing it as a behavioural form of habituation and, ultimately, a formal addiction (Box 1). The ICD-10 criteria for addiction clearly regard the nicotine in tobacco smoke as addicitive (World Health Organization, 1992). These criteria include compulsive use, tolerance, development of withdrawal symptoms and tendency to relapse after stopping. Furthermore, nicotine in tobacco smoke activates brain reward areas in a way comparable to that of other addictive drugs.

Less than $4 \%$ of smokers smoke less frequently than daily. The majority smoke at least 10 cigarettes per day and most light a cigarette within 30 minutes of waking. The persistent nature of nicotine use is shown by the fact that $48 \%$ of smokers in a UK study had been unable to abstain from smoking for any period in excess of 1 week in the previous 5 years (Russell, 1986). In the same study, craving was reported in $47 \%$ of smokers following attempts to stop. Stolerman \& Jarvis (1995) report that over 50\% of smokers said they wished to give up but only

Jason Luty is a specialist registrar on the Maudsley Hospital general psychiatry rotation. He has published on addictions and has been involved with the Maudsley Hospital Smoker's Cessation Clinic. His research interests include pharmacology and the molecular mechanisms of receptor desensitisation and tolerance. Correspondence address: Laurel House, 2 Blean Grove, Penge, London SE20 8QU, UK). The author has no pecuniary interest in the use of smoking cessation products. 
$13 \%$ thought they were likely to succeed. Only about $2 \%$ of smokers quit per year without professional advice or help. Furthermore, less than $20 \%$ of those embarking on an intensive treatment aimed at abstinence succeed for over 1 year. To put these facts into perspective, among smokers who suffered a heart attack, $40 \%$ return to smoking while still in hospital and $50 \%$ of those who have undergone surgery for lung cancer resume the habit. These facts clearly indicate a compulsion (an irresistible urge) to smoke.

Numerous studies also show a clear withdrawal syndrome in human subjects (Benowitz, 1988). The symptoms include irritability, poor concentration, anxiety, restlessness, increased hunger, depressed mood and craving for tobacco (Hughes, 1992). Symptoms develop within 12 hours and can persist for 3 weeks, although appetite is increased for over 10 weeks (Benowitz, 1988). These symptoms also occur to some degree after withdrawal of nicotine replacement therapy (NRT) or smokeless tobacco. This suggests that symptoms represent a physiological withdrawal from nicotine rather than a behavioural response to the process of smoking cigarettes.

A large number of studies have shown that tolerance to nicotine develops, particularly to its adverse effects such as nausea (Swedberg et al, 1990). Tolerance is reported in humans within days of starting to smoke.

Finally, the relapse rate among smokers who quit is comparable to rates in heroin addicts and alcoholics (Hughes, 1992).

\section{Pharmacology of nicotine in tobacco smoke}

Nicotine is a tertiary amine whose levorotatory isomer produces the majority of its physiological effects. This is the predominant isomer present in tobacco smoke. Nicotine is a weak base and can readily cross cell membrane and the blood-brain barrier at physiological $\mathrm{pH}$. It is an agonist at the nicotinic acetylcholine receptors of the autonomic ganglia, adrenal medulla and neuromuscular junction and nicotinic receptors in the brain (Benowitz, 1988). Nicotine causes an acute increase in blood pressure and heart rate, although rapid tolerance to these effects develops. Nicotine also increases circulating catecholamine levels. It causes a reduction in body weight of $4-5 \mathrm{~kg}$. These effects occur at the plasma nicotine concentrations to which smokers are exposed.

In the UK, a typical smoker consumes 14-17 cigarettes per day (Stolerman \& Jarvis, 1995). Each cigarette provides around $1 \mathrm{mg}$ of nicotine. An average smoker inhales $10-80 \mathrm{mg}$ of nicotine per day to maintain a plasma concentration of 10 $50 \mathrm{ng} / \mathrm{ml}$. Nicotine is distilled from the tobacco burning on tar droplets that are inhaled and deposited in small airways and alveoli. This allows even more rapid absorption through the lungs than occurs with intravenous administration. Smoking a single cigarette produces peak increments of plasma nicotine concentrations of $5-30 \mathrm{ng} / \mathrm{ml}$ within minutes. Hence, cigarettes are excellently designed methods of rapidly administering and adjusting plasma nicotine concentrations. Smokers appear to be able to regulate the plasma concentration of nicotine on a puff-by-puff basis depending on the type of tobacco and rate and depth of puffing. Unfortunately, so-called low-tar cigarettes appear to deliver a dose of nicotine similar to that of other cigarettes because smokers inhale more deeply when smoking them (Benowitz, 1988).

Nicotine has a half-life of 2 hours and is eliminated by direct renal excretion and metabolism to cotinine and nicotine- $N$-oxide. Nicotine therefore accumulates during the early part of the day and is almost completely eliminated overnight. Hence, the majority of smokers are beginning to suffer withdrawal symptoms on waking. (Incidentally, cotinine in urine or saliva is commonly used to monitor compliance with smoking cessation programmes.)

Laboratory studies in rats, monkeys, dogs and humans clearly show the rewarding effects of nicotine (Goldberg et al, 1981). Animal studies also suggest that its effects more closely resemble the effects of stimulants (amphetamine and cocaine) than they do the effects of other addictive drugs (sedatives or opiates). In humans who smoke, nicotine has been shown to improve attention, learning, reaction times, problem-solving and behavioural arousal (Henningfield, 1984). However, this appears to be primarily due to relief from withdrawal states. Nicotine administration in dependent animals produces release of dopamine in the nucleus accumbens, which is part of the putative reward pathway that is activated by most addictive drugs, including alcohol, opiates and stimulants (Stolerman \& Jarvis, 1995).

\section{Smoking cessation}

The UK Government has made smoking cessation one of its top 13 priorities. The Royal College of Physicians published smoking cessation guidelines (Raw et al, 1998) and these have been recently updated (West et al, 2000).

General measures to encourage and assist smoking cessation are the mainstay of NHS smoking 


\section{Box 2 General measures to encourage smoking cessation: the four $A$ s}

Ask about smoking at least once each year

Advise smokers to stop and refer to specialist services if possible

Assist smokers to stop (e.g. by referral to a specialist clinic and by providing nicotine replacement therapy or bupropion)

Arrange follow-up if appropriate

\section{Box 4 Common features of smoking} cessation programmes

First group is held 1-2 weeks prior to quit date Groups meet for 1-hour weekly sessions over 6 weeks

Groups include 15-25 participants

Instruction is given on nicotine replacement therapy and use of bupropion

Group members encourage and support each other in their attempt to quit cessation treatment (Box 2). General practitioners (GPs) and all health professionals are asked to give brief opportunistic advice to all smokers. Those who express any interest in stopping should then be referred to the specialist services that are now being set up (West et al, 2000). GPs should aim to advise all smokers to stop, and record having done so, at least once a year. Primary care teams should also ensure that their records are kept up to date concerning the smoking status of their patients.

Assistance to stop smoking is best provided by a specialist smoking cessation clinic or full-time dedicated health professional. GPs should reinforce this advice or provide such assistance in the absence of a dedicated service. It takes around 5 minutes to give basic advice such as that shown in Box 3 .

The specialist smoking cessation services being set up are intended both to treat all smokers who wish to quit and to train other professionals to provide brief interventions. West et al's (2000) guidelines indicate that specialist services should be set up in each health authority (typically serving 500000 people by providing a throughput of around 1500 clients per year). It is preferable to provide either a central smoking cessation clinic or, in rural areas, arrange for a peripatetic service to visit local clinics over a wider locality. Specialist clinics and other support services should be staffed by individuals specially trained and employed for the purpose rather than attempting to fit the job in with other duties. They may come from a variety of professional groups, including nurses and pharmacists.

\section{Box 3 Advice to help people stop smoking}

Choose a 'quit' day

Use nicotine replacement therapy (it is safer than continued exposure to the nicotine in cigarettes) or bupropion

Review what may have caused past relapses Enlist the help of family and friends
Smoking cessation clinics usually work with groups of clients, although individual treatment is also used. The format of smoking cessation programmes varies, but common features are shown in Box 4. A group meeting held in the 1- or 2-week gap prior to the quit date enables clients to start bupropion or obtain NRT in advance of the quit date. The first week proper is introductory and smokers are expected to stop smoking completely after this session. NRT is distributed and discussed. All tobacco, cigarettes, lighters and other smokingrelated products are disposed of before or during this session. Subsequent sessions concentrate on the group discussion of members' problems with quitting and on encouraging each other. This groupcentred motivational approach may work better than didactic teaching by therapists, although this has yet to be confirmed in controlled trials. Groups typically work well with 15-25 participants and two therapists (Hajek et al, 1985). At the start of each meeting, expired carbon monoxide is measured using commercial hand-held devices, to test for abstinence. At the end of the course, follow-up sessions can be offered at intervals up to 12 months.

\section{Bupropion}

Bupropion is an atypical antidepressant that inhibits neuronal reuptake of both dopamine and noradrenaline (Asher et al, 1995). It is unclear whether its anti-smoking action is related to its antidepressant activity. However, some research shows that bupropion is as effective in smokers both with and without depression (Hughes et al, 1999). It has been licensed in the USA as an antidepressant since 1989, where anecdotal reports suggested that it might help patients to stop smoking. A slow release preparation has recently been licensed in the UK for use in smoking cessation. This has been found to be at least as effective as NRT in placebo-controlled double-blind studies involving 1500 smokers (Jorenby et al, 1999). 
Box 5 Forms of nicotine replacement therapy

Chewing-gum

Transdermal patches

Inhalators

Nasal sprays

Sublingual microtabs

Lozenges

Treatment with bupropion should begin while the patient is still smoking, typically within 2 weeks of the proposed quit date. A 7- to 12-week course of bupropion is recommended. Side-effects are generally mild and typical of antidepressants (dry mouth, headache and insomnia). There is also a small risk of inducing seizures (1 in 1000) (Rosentien et al, 1993). Bupropion treatment currently costs $£ 85.70$ for a 2-month course, although it is only available on prescription and therefore a user in the British NHS would only pay the appropriate prescription charge.

It may be possible to administer both NRT and bupropion together, although only limited information is available on such combinations. Weekly blood pressure measurements are recommended in this situation. There appears to be no scientific reason to avoid the combined use of two or more different NRT formulations or NRT products with bupropion.

\section{Nicotine replacement therapy}

All health professionals should know that NRT is now available on the NHS. It is recommended that smokers use NRT for up to 3 months after quitting. Box 5 summarises the forms in which NRT can be provived. Six such formulations are available in the UK both on prescription and as over-the-counter medications (Anonymous, 1999).

Nicotine chewing-gum releases nicotine over 30 minutes, and it enters the bloodstream through the oral mucosa. Chewing-gum is available in 2 and $4 \mathrm{mg}$ formulations, and no more than 15 pieces should be chewed each day. It may be more effective for heavier smokers (i.e. more than 20 cigarettes a day) to use the $4 \mathrm{mg}$ formulations. Nicotine microtabs provide sublingual release of nicotine. These are also available in 2 and $4 \mathrm{mg}$ formulations. Up to $80 \mathrm{mg}$ can be used daily. Sugar-free mint lozenges have recently been introduced, containing $1 \mathrm{mg}$ of nicotine. Up to 25 lozenges can be used each day. Transdermal nicotine patches release nicotine for 16-24 hours. They can be placed anywhere on the body, although it is sensible to vary the site each day and also to apply the patches to areas of skin where they are less likely to be rubbed off. Patches are usually applied or changed first thing in the morning. Nicotine inhalators contain a cartridge that releases nicotine vapour when the patient sucks on the device. The nicotine is absorbed through the oropharyngeal mucosa rather than the lungs. Up to 12 cartridges can be used each day, although six are usually sufficient. All of these NRT formulations produce a peak blood nicotine concentration after 20-30 minutes. Nicotine nasal sprays are used in each nostril, which gives rapid absorption through the nasal lining. With nasal sprays, blood nicotine concentration reaches a peak after 10-15 minutes, and they can be used every 30 minutes.

None of the available NRT formulations produce a peak nicotine concentration as rapidly as that following smoking. Although replacement therapy can partially prevent withdrawal symptoms, none of the currently available nicotine replacement products (perhaps with the exception of the nasal spray) give sufficiently rapid absorption to have an acute effect that is subjectively rewarding (Hughes et al, 1994). This may partly explain the relatively high failure rates of NRT in smoking cessation. NRT approximately doubles cessation rates; the proportion of clients who remain abstinent at 6 months is raised from $11 \%$ following brief advice from clinicians to $18 \%$ with the addition of NRT, regardless of the setting (Anonymous, 1999).

The manufacturers of several NRT products also provide telephone counselling services and advice regarding various other psychosocial supports. However, the uptake rates for these helplines is very low and their value is therefore uncertain (Lando et al,1997). Nevertheless, many randomised controlled trials have shown that psychosocial support significantly enhances quit rates when compared to NRT alone (see Effectiveness of treatment, below).

\section{NRT in patients with medical problems}

Every hospital should have a dedicated specialist counsellor to advise hospital patients who smoke and wish to quit. NRT products have not been shown to increase rates of heart disease and hypertension or to exacerbate other cardiovascular disorders (Benowitz, 1998). Nevertheless, there has been some concern expressed about the routine use of NRT by people who have medical problems such as those listed in Box 6. Although NRT contains nicotine as an essential ingredient, several data sheets clearly state that 
Box 6 Relative cautions for the use of nicotine replacement therapy

Cardiovascular disease

Gastritis

Peptic ulcer disease

Systemic hypertension

Diabetes mellitus

Hyperthyroidism

Phaeochromocytoma

Renal or hepatic insufficiency

Note: NRT presents a lesser hazard than smoking

"Although nicotine's cardiovascular effects may be deleterious to patients with a history of coronary artery disease, [NRT] presents a lesser hazard than smoking which introduces carbon monoxide as an additional toxic factor." (Association of the British Pharmaceutical Industry; ABPI, 2000)

Smoking introduces over 200 additional compounds into the body, many of which are both atherogenic, thrombogenic and carcinogenic (Benowitz, 1998). Furthermore, the blood levels of nicotine produced by NRT are typically lower than those produced by smoking (Oates \& Wood, 1988).

These facts indicate that it is certainly in a patient's best interest to use NRT to stop smoking, even in the presence of certain medical conditions, assuming that they cannot stop smoking without nicotine replacement. There have been at least five cases in the USA where smokers on NRT suffered serious cardiac events. However, in these cases investigations by the US Food and Drug Administration determined that NRT was not responsible (Benovitz \& Gourlay, 1997). The most recent smoking cessation guidelines for health professionals state that

"NRT can be recommended for use in patients with cardiovascular disease but only with the agreement of the patient's physician if the disease is acute or poorly controlled." (West et al, 2000)

\section{NRT and dependence}

Early trials suggested that up to one-third of smokers who quit use NRT for 1 year. However, this figure is probably much too high and, in practice, very few smokers who quit use NRT for longer than the time suggested on the product label. Nevertheless, abrupt cessation of NRT can produce nicotine withdrawal symptoms (Hughes, 1989). These facts have raised worries that NRT is itself addictive. However, the
ABPI advises that NRT rarely leads to dependence, and if it does the dependence is less harmful and easier to break than smoking dependence (ABPI, 2000).

\section{Smoking cessation in pregnancy}

Specialist counsellors should be available to advise pregnant smokers who wish to quit. The safety of NRT and bupropion have not been established in pregnancy. As with most other medications, manufacturers are reluctant to licence the use of these products in pregnancy. Smoking exposes the foetus to much higher peak levels of nicotine than NRT does and also introduces many other potential teratogens such as carbon monoxide. Current recommendations are to give "firm and clear advice to stop smoking throughout pregnancy, and give assistance when it is required" (Parrott et al, 1998). Unfortunately, there is no clear advice regarding whether pharmacotherapy should be used or not to help pregant women quit. However, current guidelines do state that use of NRT in pregnancy may benefit the foetus and the mother if it leads to smoking cessation (West et al, 2000).

\section{Effectiveness of treatment}

At least 80 randomised controlled trials have studied smoking cessation therapies using NRT, and many other trials have analysed the effects of alternative forms of psychosocial treatments. Expert committees, including the Cochrane Tobacco Addiction Review Group in the UK and its US equivalent, the Agency for Health Care Policy Research, have concluded that treatment for smoking cessation is effective. Overall, smoking cessation treatments in the UK cost under $£ 1000$ per life-year saved. Even the most intensive smoking cessation treatment, involving specialist clinics, NRT and behavioural advice, costs about $£ 873$ per life-year saved. By comparison, the median cost to the NHS of life-saving treatment and interventions for continuing smokers is around $£ 17000$ per life-year saved (Parrott et al, 1998).

Brief advice from GPs increases abstinence rates at 6 months by $1-3 \%$ above control levels. This advice should be given to all smokers every year, whether or not they wish to quit. It has been estimated that $40 \%$ of smokers make some attempt to quit in response to advice from a GP. Overall, this would 
take up 20-40 hours of GP time each year (about 8 hours of GP time would be required per ex-smoker created). This translates to a cost of $£ 174$ per lifeyear saved (Parrott et al, 1998).

Specialist smoking cessation clinics can increase long-term (1-year) quit rates by $17 \%$ above control levels by combining behavioural and pharmacological techniques in those who are motivated to attend (West et al, 2000). Controlled trials suggest that intense psychosocial treatments are more effective than low-intensity support (such as routine care by a GP). However, NRT and bupropion approximately doubled cessation rates in placebocontrolled trials in several different treatment settings, regardless of the form of NRT and the nature, if any, of additional psychosocial measures (Anonymous, 1999).

\section{References}

Anonymous (1999) Nicotine replacement to aid smoking cessation. Drug and Therapeutics Bulletin, 37, 52-54.

Association of the British Pharmaceutical Industry (2000) ABPI Data Sheet Compendium 1999/2000. London: $\mathrm{ABPI}$

Asher, J. A., Coles, J. O. \& Colin, J.-N. (1995) Bupropion: a review of its antidepressant activity. Journal of Clinical Psychiatry, 56, 395-401.

Benowitz, N. L. (1988) Pharmacologic aspects of cigarette smoking and nicotine addiction. New England Journal of Medicine, 319, 1318-1330.

- (1998) Nicotine Safety and Toxicity. Oxford: Oxford University Press.

- \& Gourlay, S.G.G. (1997) Cardiovascular toxicity of nicotine: implications for nicotine replacement therapy. Journal of the American College of Cardiology, 29, 1422-1431.

Callum, C. (1998) The Smoking Epidemic. London: Health Education Authority.

Goldberg, S. R., Spealman, R. D., \& Goldberg, D. M. (1981) Persistent behaviour at high rates maintained by intravenous self-administration of nicotine. Science, 214, 573-575.

Hajek, P., Belcher, M. \& Stapleton, J. (1985) Enhancing the impact of groups. British Journal of Clinical Psychology, 24, 289-294.

Henningfield, J. E. (1984) Behavioural pharmacology of cigarette smoking. In Advances in Behavioural Pharmacology (eds T. Thompson, P. B. Dews \& J. E. Barrett), pp. 131210. New York: Academic Press.

Hughes, J. R. (1989) Dependence potential and abuse liability of nicotine replacement therapies. Biomedical Pharmacotherapy, 43, 11-17.

- (1992) Tobacco withdrawal in self-quitters. Journal of Consulting Clinical Psychology, 60, 689-697.

- Higgins, S. T. \& Bickel, W. K. (1994) Nicotine withdrawal versus other drug withdrawal syndromes. Addiction, 89 1461-1470.

_, Goldstein, M. G., Hurt, R. D., et al (1999) Recent advances in pharmacotherapy of smoking. Journal of the American Medical Association, 281, 72-76.

Jorenby, D. E., Leischow, S. J. \& Mides, M. A. (1999) A controlled trial of sustained-release bupropion, a nicotine patch, or both for smoking cessation. New England Journal of Medicine, 340, 685-691.

Lando, H. A., Rolnick, S., Klevan, D., et al (1997) Telephone support as an adjunct to transdermal nicotine in smoking cessation. American Journal of Public Health, 87, 1670-1674.
Oates, J. A. \& Wood, A. J. J. (1988) Pharmacologic aspects of cigarette smoking and nicotine addiction. New England Journal of Medicine, 319, 1318-1330.

Office for National Statistics (2000) Results from the 1999 General Household Survey. London: HMSO.

Parrott, S., Godfrey, C., Raw, M., et al (1998) Guidance for commissioners on the cost effectiveness of smoking cessation interventions. Thorax, 53 (suppl. 5), S2-S37.

Peto, R., Lopez, A. D. \& Boreham, J. (1994) Mortality from Smoking in Developed Countries, 1950-2000. Oxford: Oxford University Press.

Raw, M., McNeill, A. \& West, R. (1998) Smoking cessation guidelines for health professionals - a guide to effective smoking cessation interventions for the health care system. Thorax, 53 (suppl. 5), S1-S18.

Rosentien, D. L., Nelson, C. \& Jacobs, S. C. (1993) Seizures associated with anti-depressants. Journal of Clinical Psychiatry, 54, 289-296.

Russell, M. A. H. (1986) Conceptual framework for nicotine substitution. In The Pharmacologic Treatment of Tobacco Dependence (ed. J. K. Ockene), pp. 90-107. Cambridge, MA: Harvard University Press.

Stolerman, I. P. \& Jarvis, M. J. (1995) The scientific case that nicotine is addictive. Psychopharmacology, 117, 2-10.

Swedberg, M. D. B., Henningfield, J. E. \& Goldberg, S. R. (1990) Nicotine dependency: animal studies. In Nicotine Psychopharmacology: Molecular, Cellular and Behavioural Aspects (eds S. Wonnacott, M. A. H. Russell \& I. P. Stolerman), pp. 38-76. Oxford: Oxford Science Publications.

West, R., McNeill, A. \& Raw, M. (2000) Smoking cessation guidelines for health professionals. Thorax, 55, 987-999.

World Health Organization (1992) The ICD-10 Classification of Mental and Behavioural Disoders. Geneva: WHO.

\section{Multiple choice questions}

1. With regard to smoking in the UK:

a around 120000 people die each year of smoking-related diseases

b $25 \%$ of smokers will die of smoking-related diseases

c a continuing smoker has a life-expectancy 8 years less than a non-smoker

d UK government revenues from tobacco vastly exceed the costs of treating smoking-related diseases

e low-tar cigarettes are much safer than other cigarettes.

2. Smoking typically produces the following addictive effects:

a craving during periods of abstinence

$\mathrm{b}$ a withdrawal syndrome including poor concentration

c tolerance to adverse effects

d a withdrawal syndrome lasting about 1 week

e persistent use despite evidence of harm.

3. Regarding smoking cessation treatment:

a this is now a top UK government priority for improving the nation's health

b only specialised smoking cessation clinics should encourage people to stop smoking 
c intensive smoking cessation treatments are more cost-effective than brief advice

d smoking cessation treatment is cost-effective for the NHS

e in smoking cessation clinics, group-centred motivational approaches may work better than didactic teaching from therapists.

4. Nicotine replacement therapy:

a can be obtained without a prescription

b doubles cessation rates

c works only for light-smokers

$\mathrm{d}$ is proven to be superior in effectiveness than bupropion

e should only be used in conjunction with other behavioural support programmes.

5. Nicotine replacement therapy:

a should never be used in patients with cardiovascular disease

b can be used in pregnancy c causes cancer if used for a protracted period

d avoids the smoker being exposed to over 200 damaging compounds in tobacco smoke

e completely eliminates withdrawal symptoms in most smokers.
MCQ answers

$\begin{array}{lllll}1 & 2 & 3 & 4 & 5\end{array}$

a $\mathrm{T}$ a $\mathrm{T}$ a $\mathrm{T}$ a $\mathrm{T}$ a $\mathrm{F}$

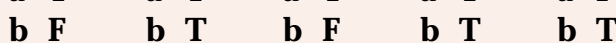

c $T$ c $T$ c c $F \quad$ c $F \quad$ c $F$

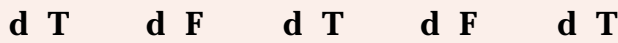

$\begin{array}{llllllll}\text { e } F & \text { e } T & \text { e } T & \text { e } F & \text { e } F\end{array}$

\section{Coming soon from Gaskell}

\section{Use of drugs in psychiatry: the evidence from psychopharmacology (5th edn)}

By John Cookson, Cornelius Katona and David Taylor

A fully revised and updated edition of this invaluable text covers all aspects of drug treatment in psychiatry. The authors have combined their wealth of knowledge and clinical experience with the best evidence from research to produce a book that will be useful for all clinical psychiatrists.

- In-depth coverage of all drugs currently prescribed for psychiatric indications

- Handy, pocket-book format with quick-reference features

- Comprehensive introduction to psychopharmacology, ideal for the trainee

- Detailed clinical effectiveness and 'number needed to treat' data included

February 2002, ISBN 190124229 3, £20.00

Contact: Book Sales, The Royal College of Psychiatrists,

17 Belgrave Square, London, SW1X 8PG, UK

Tel: +44 (0)20 72352351 ext 146. Fax: +44 (0)20 7245 1231. Website: $h t t p: / / w w w . r c p s y c h . a c . u k$ 